\title{
Efectos de fatiga en variables cinemáticas y cinéticas de miembros inferiores en jugadores de fútbol
}

\author{
$\checkmark$ Laura Andrea Quintero Palma ${ }^{1}$ \\ WiLFREDo AGREDo ${ }^{2}$ \\ JESSICA QUiCENO HENAO ${ }^{1}$ \\ ALEXANDER QUIÑNONES ${ }^{1}$ \\ JosÉ FERNANDO TOVAR ${ }^{3}$
}

\section{Resumen}

En las lesiones más comunes, especialmente en deportistas, están involucrados los miembros inferiores, siendo las rodillas las más afectadas a diferencia los tobillos y cadera como lo manifiesta la FIFA. La lesión del Ligamento Cruzado Anterior (LCA) causa mayor gasto financiero y un mayor número de días perdidos en el deporte. Los mecanismos de lesión se dan por contacto y no contacto, siento la segunda una de las más estudiadas e investigadas ya que pueden prevenirse para evitarla. Además, se ha encontrado que la mayoría de las lesiones ocurren en las últimas etapas de la competencia, lo que indica que el ejercicio vigoroso y la fatiga pueden influir la ocurrencia. El objetivo es determinar los efectos de la fatiga en la biomecánica de jugadores de fútbol, para tener una aproximación y descripción de estas variables de esta población de estudio. Dentro de los resultados principales se obtuvo que la flexión de rodilla fue principalmente la variable más afectada después de la fatiga en las maniobras de corte, pivote y aterrizaje en una sola pierna. Los cambios observados en los momentos de fuerza de la rodilla no fueron significativos, tal como están descritas en la literatura como factores de riesgo relacionados con la biomecánica de la rodilla y con esta lesión en deportistas jóvenes. De las variables cinéticas no tuvieron mayor efecto después del protocolo..

Palabras clave: Fatiga, fútbol, lesión, ligamento cruzado anterior.

\footnotetext{
Institución Universitaria Escuela Nacional del Deporte. Cali, Colombia.

Universidad Autónoma de Occidente. Cali, Colombia.

2 Universidad del Valle. Cali, Colombia.
}

Autor de correspondencia: Quintero Palma, L.A. (Laura Andrea): Institución Universitaria Escuela Nacional del Deporte - Facultad de Ciencias de la Educación y del Deporte: Calle 9 Nro.34-01. Correo electónico: laura.quintero@ endeporte.edu.co
Historia del artículo:

Artículo recibido: 29-X-2019/ Aprobado: 15-I-2020

Disponible online: 15 de abril de 2020

Discusión abierta hasta septiembre de 2021 


\section{Effects Of Fatigue On Kinematic And Kinetic Variables Of Lower Limbs In Soccer Players}

\section{Abstract}

In the most common injuries, especially in athletes, the lower limbs are involved, with the knees being the most affected, unlike the ankles and hips, as stated by FIFA. The injury of the Anterior Cruciate Ligament (ACL) causes greater financial expense and a greater number of days lost in the sport. The mechanisms of injury are given by contact and not contact, I feel the second one of the most studied and investigated since they can be prevented to avoid it. In addition, it has been found that most injuries occur in the last stages of competition, indicating that vigorous exercise and fatigue can influence the occurrence. The objective is to determine the effects of fatigue on the biomechanics of soccer players, to have an approximation and description of these variables of this study population. Among the main results it was obtained that knee flexion was mainly the most affected variable after fatigue in the maneuvers of corte, pivote and landing on one leg. The changes observed in moments of knee strength were not significant, as described in the literature as risk factors related to biomechanics of the knee and with this injury in young athletes. Of the kinetic variables they had no greater effect after the protocol.

Keywords: Fatigue, soccer, injury, anterior cruciate ligament.

\section{Efeitos da fadiga nas variáveis cinemáticas e cinéticas de membros inferiores em jogadores de futebol}

\section{Resumo}

Nas lesões mais comuns, principalmente em atletas, os membros inferiores estão envolvidos, sendo os joelhos os mais afetados, diferentemente dos tornozelos e quadris, como afirma a FIFA. A lesão do ligamento cruzado anterior (LCA) causa maior despesa financeira e maior número de dias perdidos no esporte. Os mecanismos de lesão são dados por contato e não por contato, sinto o segundo dos mais estudados e investigados, pois podem ser evitados para evitá-lo. Além disso, verificou-se que a maioria das lesões ocorre nos últimos estágios da competição, indicando que exercícios vigorosos e fadiga podem influenciar a ocorrência. 0 objetivo é determinar os efeitos da fadiga na biomecânica de jogadores de futebol, para ter uma aproximação e descrição dessas variáveis da população estudada. Dentre os principais resultados, obteve-se que a flexão do joelho foi principalmente a variável mais afetada após fadiga nas manobras de corte, rotação e aterrissagem em uma perna. As alterações observadas nos momentos de força do joelho não foram significativas, conforme descrito na literatura como fatores de risco relacionados à biomecânica do joelho e com esta lesão em atletas jovens. Das variáveis cinéticas, elas não tiveram maior efeito após o protocolo.

Palavras-chave: Fadiga, futebol, lesão, ligamento cruzado anterior.

\section{Introducción}

Las lesiones deportivas perjudican significativamente el rendimiento de un deportista y de su equipo en general. Por lo tanto, la prevención de estas se convierte en una prioridad desde la investigación científica (Hägglund et al., 2013). La mayoría de los estudios que examinan los efectos del ejercicio sobre las habilidades futbolísticas, han evaluado el dominio técnico antes y después del ejercicio físico ((Ali et al., 2007), Ostojic et al., 2202)) 6-8. Además, estudios previos han empleado protocolos de ejercicio que no son representativos de las demandas de partido porque no incluyen patrones de movimiento específicos de fútbol (Ali et al., 2007). 
En consecuencia, existe poca evidencia para evaluar los efectos específicos del fútbol después de la fatiga sobre habilidades realizadas durante el juego. Una de las lesiones más comunes en deportes es la de Ligamento Cruzado Anterior (LCA) (Parra, 2014). Existen dos mecanismos de lesión para este ligamento, se puede producir por contacto o sin contacto. El mecanismo de lesión de ésta es generalmente por no contacto, que significa, que no hay contacto físico sobre la rodilla cuando la lesión ha ocurrido y es una de las más frecuentes, hasta un 70\% de las lesiones (Zahínos et al. 2010). En un estudio retrospectivo evaluaron las lesiones de LCA durante la actividad deportiva, se reportaron que el $65 \%$ de las lesiones fueron por no contacto, todas ellas cuando la rodilla se encontraba cerca a la extensión y con un valgo dinámico excesivo. De esto se puede decir que la naturaleza de este mecanismo de lesión puede ser reducida a través de un entrenamiento neuromuscular apropiado con el fin de modificar la biomecánica de miembros inferiores en tareas deportivas, especialmente en los aterrizajes y cambios de dirección. (Boden et al., 2007).

Aunque las jugadoras de fútbol femenino son por lo menos dos veces más propensas a lesiones del ligamento cruzado anterior, los jugadores de fútbol masculinos que sufren lesiones del LCA siguen siendo frecuentes en el deporte. De los 256 millones de jugadores de fútbol en el mundo, el 90\% de ellos son hombres. No obstante, la identificación de los mecanismos de lesión del LCA y el riesgo se han investigado principalmente en las mujeres, por lo cual existe una necesidad crítica de determinar el mecanismo de lesión y los factores de riesgo subyacentes para las lesiones del LCA en jugadores de fútbol masculinos.

La lesión del LCA causa el mayor gasto financiero y el mayor número de días perdidos del jugador en el deporte. En los desgarres de LCA sin contacto, los jugadores de fútbol masculinos son más propensos a lesionar su rodilla derecha (74\%) en comparación con la rodilla izquierda, aunque depende de la dominancia del deportista (Brophy, 2010). Las situaciones comunes de juego asociadas con la lesión del LCA sin contacto incluyen: cambio de dirección, aterrizar después de un salto (después de encabezar la pelota), y girar con un pie apoyado en el suelo, generando efecto pivote de la pierna apoyada. Se ha descubierto que la mayoría de las lesiones ocurren en las últimas etapas de la competencia, lo que indica que el ejercicio vigoroso y la fatiga pueden influir en los factores biomecánicos (Rodacki, 2001). Se sabe que en un estado de fatiga los músculos que rodean la articulación de la rodilla pueden perder la capacidad de proteger la articulación. El cambio neuromuscular alterado debido a la fatiga puede reflejarse por cambios en la cinemática de las articulaciones, magnitudes verticales de la fuerza de reacción en el suelo (cinética) y la actividad electromiográfica (EMG). Además, estos cambios también se pueden observar como una alteración de la rigidez de las extremidades inferiores durante el aterrizaje (Padua et al., 2006). Sin este conocimiento, las intervenciones para entrenar y prevenir lesiones del LCA probablemente seguirán siendo sólo parcialmente efectivas. La hipótesis de esta investigación es que el protocolo de fatiga dará lugar a cambios en las variables cinemáticas y cinéticas de riesgo de lesión de LCA en los miembros inferiores en jugadores de fútbol.

El objetivo de esta investigación es determinar los efectos de la fatiga en la biomecánica de jugadores de fútbol, lo cual servirá para tener una aproximación de las variables cinemáticas y cinéticas de esta población de estudio y relacionarlas con el riesgo de presentar la lesión al llevar a la fatiga al deportista, el protocolo de fatiga implementado fue el SAFT 90 (Barrett et al., 2013) este simula las demandas fisiológicas y patrones de movimientos específicos de la práctica deportiva. Los sujetos fueron incluidos en el estudio si no han tenido una lesión ni antecedentes quirúrgicos en las extremidades inferiores y si no presentan alteraciones en el equilibrio.

\section{Materiales y Métodos}

\section{Sujetos}

En la investigación participaron 30 jugadores masculinos de fútbol de categoría sub 17 pertenecientes a un club deportivo de la región, se hizo extensa la invitación a participar de manera escrita a diferentes clubes, obteniendo respuesta y disponibilidad de evaluación de solo un club. Los sujetos cuentan con edades entre 14 y 20 años, con peso entre 53 y $80 \mathrm{~kg}$ ( 2 futbolistas con peso superior a $79 \mathrm{~kg}$, arquero y volante), se obtuvo un peso promedio de $67.3 \mathrm{~kg}$, en cuanto a la estatura se tienen futbolistas con alturas entre 164.6 y 193.4 
cm. A todos los participantes se les proporcionó un consentimiento informado por escrito de acuerdo con los procedimientos éticos de la institución universitaria donde se desarrolló la investigación.

\section{Materiales y equipos}

El movimiento en tres dimensiones de los marcadores ubicados en los miembros inferiores se recolectaron mientras realizaban las maniobras utilizando cinco cámaras optoelectrónicas Qualisys modelo Oqus 5 (Gothemburg, Sweden) con una frecuencia de muestreo de $150 \mathrm{~Hz}$, por medio del software Qualisys Track Manager (QTM versión 19.2; Qualisys) se realizó el procesamiento inicial de los datos de las trayectorias, la identificación y llenado de gaps en la señal de cada marcador y para el procesamiento e integración de datos cinemáticos y cinéticos por medio del software Visual 3D (C-motion Biomechanics Software, Inc) . Las fuerzas de reacción de la tierra (GRFs) se recolectaron de una plataforma de fuerza AMTI (modelo No. 600600; Advanced Mechanical Technology Inc) empotrada al suelo del laboratorio con una frecuencia de muestreo de $1000 \mathrm{~Hz}$.

\section{Procedimiento Experimental}

Este estudio fue desarrollado en un laboratorio de biomecánica con un grupo de deportistas sanos ejecutando maniobras típicas en fútbol, se midieron variables cinemáticas y cinéticas asociadas al riesgo de lesión del ligamento cruzado anterior, se tuvo en cuenta la dominancia de cada uno y los años de experiencia de su práctica deportiva.

\section{Maniobras}

Todos los participantes realizaron tres maniobras típicas de fútbol, con varias repeticiones cada una, de las cuales se escogieron tres que estuvieran bien realizadas. Antes de ejecutar las maniobras se realizó un calentamiento de cinco minutos. Las maniobras realizadas fueron: aterrizaje de un salto en una sola pierna (single landing leg test - SLL) desde una altura de caída de $0.3 \mathrm{~m}$, con una caja ubicada al lado de la plataforma de fuerza, corte (corte - CUT) y pivote (pivote - PIV). La tarea de SLL consistió que los participantes bajaran de una plataforma elevada y aterrizaran en una pierna en una plataforma de fuerza. Esta prueba se realizó con ambas piernas. Esta maniobra se ha citado como un mecanismo de lesión de ACL ((Jones et al., 2014), (Boden et al., 2007)). Se instruyó a cada participante para que asegurara una distancia de caída homogénea en cada prueba, al centro de la plataforma. Las pruebas en las que el participante perdía el equilibrio no se tuvieron en cuenta. La tarea de CUT (giro a $90^{\circ}$ ) implicó correr hacia una plataforma de fuerza, ubicada a 10 metros de la salida del participante (Figura 1). Luego, el participante al hacer contacto con la plataforma de fuerza con el pie derecho inmediatamente giraba a $90^{\circ}$ a la izquierda y seguía corriendo $(3 \mathrm{~m})$ hasta detenerse. Por último, para la tarea de PIV (giro $180^{\circ}$ ), los participantes se acercaron a la plataforma de fuerza de la misma manera, pero retornaron a la posición inicial original una vez que contactaron la plataforma con la pierna derecha. En ambas tareas se realizaron repeticiones para cada pierna. Se muestra el esquema de la ejecución de las maniobras las cuales permiten movimientos similares que se pueden realizar en el campo durante un partido de fútbol (Jones, 2014).

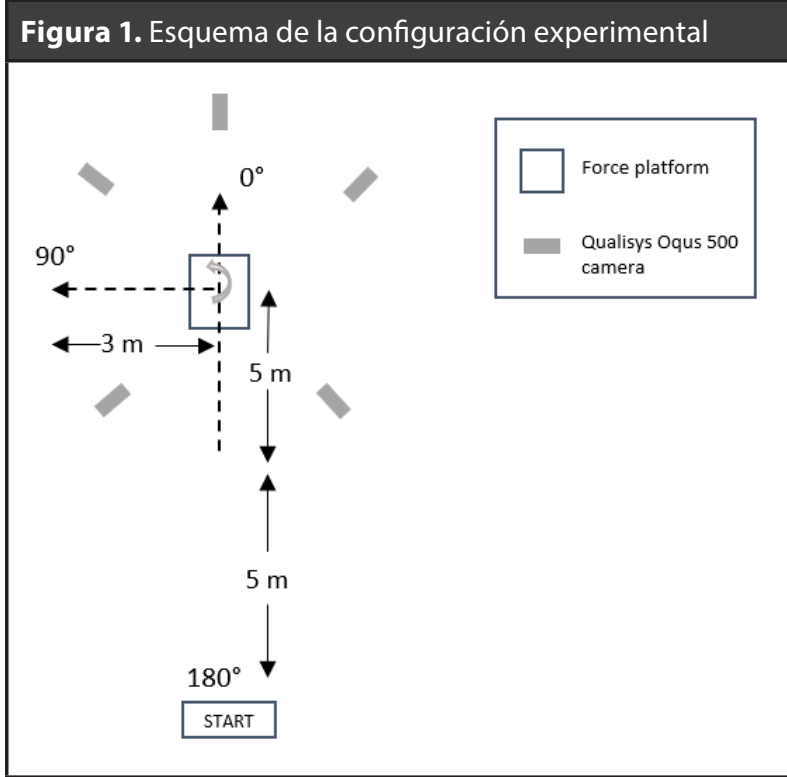

\section{Procesamiento y análisis de la información}

Marcadores reflectivos (esferas de 12,7 mm) fueron ubicados en los siguientes puntos del cuerpo para la prueba estática: espinas ilíacas anterosuperior izquierda y derecha, entre espinas ilíacas posterosuperior; dispositivo de alineación de rodilla (KAD) bilateral para la captura estática; marcadores en 
cóndilos femorales bilaterales para la captura dinámica, bandas laterales de seguimiento en los segmentos del fémur y tibia; maléolos laterales; talón y segunda cabeza metatarsiana usando cinta adhesiva de doble faz. Para las pruebas dinámicas se retiraron los KADs y se ubicaron marcadores reflectivos en los cóndilos femorales laterales respectivamente.

A partir de la prueba estática, se creó un modelo cinemático de 6 grados de libertad de la extremidad inferior para cada participante, los segmentos incluidos fueron la pelvis, el muslo, la tibia y el pie utilizando el software Visual3D (versión 3.90.21; C-Motion) (Jones, 2014). Este modelo cinemático cuantifica el movimiento en las articulaciones de cadera, rodilla y tobillo utilizando la secuencia de ángulos de Cardan x-y-z.El sistema de coordenadas local se definió en el centro de la articulación proximal para cada segmento. La posición de prueba estática fue designada como neutral de los participantes (cero anatómico). La alineación y las medidas cinemáticas subsiguientes se relacionaron con esta posición. Los momentos articulares de los miembros inferiores se calcularon utilizando dinámica inversa a través del software Visual3D.

Las pruebas de SLL para cada participante se tomaron desde el contacto inicial hasta el punto de flexión máxima de la rodilla, lo que se consideró el final de la fase de aceptación de peso para esta tarea. Se utilizaron funciones propias de Visual3D para determinar los eventos de inicio de contacto con la plataforma, y flexión máxima de la rodilla. Para las tareas de corte y pivote se consideraron de igual manera el contacto inicial, la flexión máxima de rodilla y el despegue del pie que estaba en contacto con la plataforma como eventos del movimiento. Las coordenadas de las articulaciones y los datos de momento de fuerza fueron suavizadas con un filtro digital de paso bajo Butterworth con frecuencias de corte de $12 \mathrm{Hzy} 25 \mathrm{~Hz}$, respectivamente. Las frecuencias de corte se seleccionaron basándose en un análisis residual (Winter D, 1990).

Todas las maniobras se realizaron por cada miembro inferior donde se ejecutaron tres repeticiones respectivamente. Por lo tanto, se hicieron comparaciones para las variables de ambas piernas entre las tareas. Las variables cinemáticas fueron ángulos máximos de la rodilla en los tres ejes de movimiento ya que fue necesa- rio tener un enfoque en la rodilla pues es la articulación afectada en lesión de ligamento cruzado anterior.

\section{Protocolo de fatiga}

Estudios anteriores demuestran que la fatiga durante un partido de fútbol está asociada a la disminución del control muscular, esto puede estar relacionado con mayor riesgo de presentarse lesiones a nivel de miembros inferiores observadas durante los últimos 15 min de cada mitad del juego. El protocolo fatiga (SAFT90) empleado fue desarrollado para replicar las demandas fisiológicas y mecánicas que se generan durante un partido de fútbol. Este protocolo se desarrolló sobre la base de datos contemporáneos de análisis de tiempo-movimiento. El protocolo se divide en dos períodos de 45 minutos intercedidos con un intervalo de descanso pasivo de 15 minutos. Está diseñado para incluir movimientos de utilidad multidireccional, cambios de dirección, aceleración y desaceleración frecuentes, inherentes al fútbol. Se ha validado para replicar la respuesta a la fatiga de la jugada de partido de fútbol (Small et al., 2009). El esquema se basó en una carrera de 20 m incorporando cuatro estacas de campo posicionados en las distancias que se observan en la Figura 2. Los sujetos realizan esto corriendo hacia atrás y rodeando la primera estaca, luego corriendo hacia adelante en línea recta, una vez se acerca al conjunto de tres estacas, sigue su movimiento a través de ellas, generando así cambios de dirección al pasarlas. Finalmente, continúa el recorrido hasta llegar a la posición inicial. Se desarrolló un perfil de actividad de 15 minutos y repitió seis veces durante el partido de fútbol simulado completando un total de 90 minutos. El perfil de actividad se realizó de manera aleatoria e intermitente. Para que los sujetos pudieran realizar este protocolo, se recomendó no realizar ejercicio vigoroso $24 \mathrm{~h}$ antes de la prueba y no consumir cafeína o alcohol.

Una vez realizado el protocolo completando los 90 minutos de un partido de fútbol con los 15 minutos de receso e hidratación, los deportistas retornaron al laboratorio y se ejecutaron las mismas maniobras de la evaluación PRE con el fin de volver a medir las variables biomecánicas, en las mismas condiciones controladas. 
Figura 2. Esquema de implementación del protocolo SAFT90

Fuente: Small et al., (2009) Soccer Fatigue, Sprinting and Hamstring Injury Risk

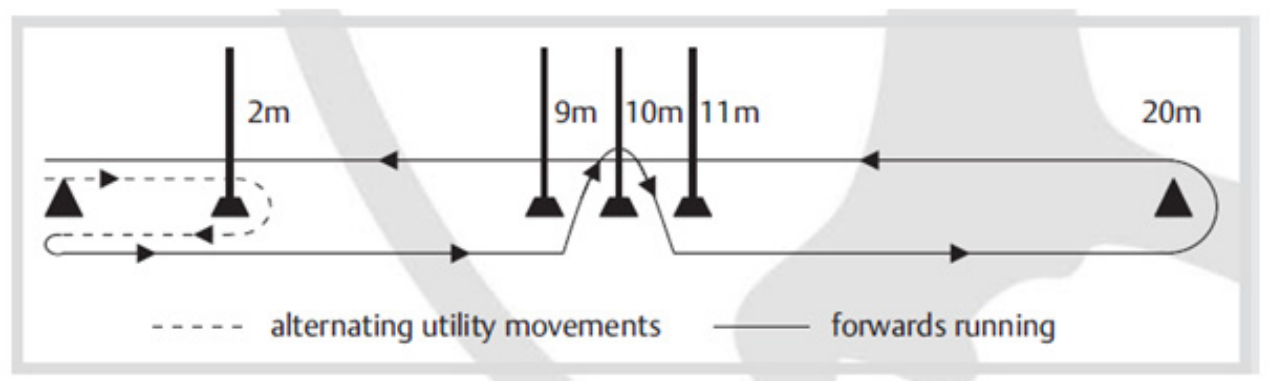

\section{Análisis estadístico}

Mediante un muestreo no probabilístico y por conveniencia se seleccionaron a 30 futbolistas, los cuales fueron sometidos a un protocolo de fatiga que simula un partido de fútbol. Los futbolistas fueron evaluados antes (procedimiento PRE) y después del protocolo (procedimiento POST). Para identificar si se presentaron cambios en las mediciones PRE y POST se realizó una prueba de hipótesis sobre las diferencias de ambas mediciones teniendo en cuenta la inferencia bayesiana; se optó por esta metodología debido a que la población objeto de estudio no presenta las condiciones de aleatoriedad necesarias para asegurar validez en los resultados con los métodos estadísticos clásicos, esto por haber hecho uso de un muestreo no probabilístico y por conveniencia, lo cual no asegura representatividad de la población. Por ende, basados en el hecho de que se puede asegurar mínimamente permutabilidad en las mediciones de los individuos, requisito necesario para la estadística bayesiana, además, dado que el tamaño de muestra es reducido, se puede ejecutar un proceso de análisis bayesiano.

Para llevar a cabo el análisis bayesiano inicialmente se debe calcular la diferencia entre las mediciones PRE y POST, en este caso particular se trabajó con el valor absoluto de cada medición, ya que el signo representa el tipo de movimiento o momento realizado. Estas diferencias se representaron por $\Delta$, dichas diferencias se calcularon como POST versus PRE.

$$
\Delta \mathrm{i}=x \text { POST } \mathrm{i}-\mathrm{xPREi} \quad i=1,2,3, \ldots, 31
$$

En este caso se asume que dichas diferencias tendrán distribución normal (Pueden ser negativas o positivas), esto es:

$$
\Delta \sim \operatorname{Normal}(\mu \Delta, \sigma 2) .
$$

Dado que se quiere saber si se presentan diferencias entre las mediciones (POST vs PRE), se plantea la siguiente prueba de hipótesis bayesiana

$$
H_{\mathrm{o}}: \mu_{\Delta}=0 \quad H_{1}: \mu_{\Delta}>0
$$

\section{Resultados}

Se inició con un análisis exploratorio para las mediciones pre y post de los 30 jugadores de fútbol. En las mediciones pre se observó que en la flexión de rodilla izquierda en la maniobra single landing leg presentó la menor variabilidad observada $(17,56 \%$, rango de baja distancia entre las mediciones) mientras que las mediciones del momento flexor de la rodilla derecha y el momento extensor de la rodilla izquierda en la maniobra corte, el momento flexor de la rodilla derecha, momento extensor de la rodilla izquierda y el momento rotador interno de la rodilla derecha realizando la maniobra pivote y el momento rotador externo de la rodilla izquierda realizando la maniobra single landing leg, presentaron la mayor variabilidad observada (superior a 52,73\%), esto se puede visualizar en la Tabla 1.

En los resultados del análisis exploratorio de las mediciones post, se encontró que las variables medidas del momento flexor de la rodilla derecha e izquierda durante la maniobra de corte, el momento flexor de la rodilla derecha e izquierda, momento rotador interno y externo de la rodilla derecha y el momento extensor de la rodilla izquierda durante la maniobra de pivote y el momento rotador externo de la rodilla derecha durante la maniobra de aterrizaje en una sola pierna presentaron la mayor variabilidad observada (superior a 52,93\%). 
TABLA 1. ANÁLISIS EXPLORATORIO DE LAS VARIABLES MEDIDAS PRE IMPLEMENTACIÓN DEL PROTOCOLO SAFT90

\begin{tabular}{|c|c|c|c|}
\hline & \multicolumn{3}{|c|}{ PRE } \\
\hline Variables & Promedio & Desviación & $\begin{array}{c}\text { Coef. } \\
\text { Variación }\end{array}$ \\
\hline $\begin{array}{c}\text { Maniobra SL leg - Articulación Rodilla - } \\
\text { Hemisferio Izquierdo - Movimiento Flexión }\end{array}$ & 41,618 & 7,308 & 17,56 \\
\hline $\begin{array}{c}\text { Maniobra Cutting - Articulación Rodilla - } \\
\text { Hemisferio Derecho - Momento Flex }\end{array}$ & 0,797 & 0,484 & 60,71 \\
\hline $\begin{array}{c}\text { Maniobra Cutting - Articulación Rodilla - } \\
\text { Hemisferio Izquierdo - Momento Ext }\end{array}$ & 0,223 & 0,125 & 55,872 \\
\hline $\begin{array}{c}\text { Maniobra Pivoting - Articulación Rodilla - } \\
\text { Hemisferio Derecho - Momento Flex }\end{array}$ & 0,856 & 0,496 & 57,996 \\
\hline $\begin{array}{c}\text { Maniobra Pivoting - Articulación Rodilla - } \\
\text { Hemisferio Derecho - Momento Rot Int }\end{array}$ & 0,181 & 0,118 & 65,152 \\
\hline $\begin{array}{c}\text { Maniobra Pivoting - Articulación Rodilla - } \\
\text { Hemisferio Izquierdo - Momento Ext }\end{array}$ & 1,038 & 0,853 & 82,238 \\
\hline $\begin{array}{c}\text { Maniobra SL leg - Articulación Rodilla - } \\
\text { Hemisferio Izquierdo - Momento Rot Ext }\end{array}$ & 0,269 & 0,142 & 52,738 \\
\hline
\end{tabular}

\section{TABLA 2. ANÁLISIS EXPLORATORIO DE LAS VARIABLES MEDIDAS POST IMPLEMENTACIÓN DEL PROTOCOLO SAFT90}

\begin{tabular}{|c|c|c|c|}
\hline & \multicolumn{3}{|c|}{ POST } \\
\hline Variables & Promedio & Desviación & $\begin{array}{c}\text { Coef. } \\
\text { Variación }\end{array}$ \\
\hline $\begin{array}{c}\text { Maniobra Cutting - Articulación Rodilla - Hemisferio } \\
\text { Derecho - Momento Flex }\end{array}$ & 0,806 & 0,525 & 65,201 \\
\hline $\begin{array}{l}\text { Maniobra Cutting - Articulación Rodilla - Hemisferio } \\
\text { Izquierdo - Momento Flex }\end{array}$ & 0,862 & 0,456 & 52,931 \\
\hline $\begin{array}{c}\text { Maniobra Pivoting - Articulación Rodilla - Hemisferio } \\
\text { Derecho - Momento Flex }\end{array}$ & 1,078 & 0,585 & 54,25 \\
\hline $\begin{array}{c}\text { Maniobra Pivoting - Articulación Rodilla - Hemisferio } \\
\text { Derecho - Momento Rot Ext }\end{array}$ & 0,27 & 0,191 & 70,801 \\
\hline $\begin{array}{c}\text { Maniobra Pivoting - Articulación Rodilla - Hemisferio } \\
\text { Derecho - Momento Rot Int }\end{array}$ & 0,264 & 0,323 & 122,109 \\
\hline $\begin{array}{l}\text { (Maniobra Pivoting - Articulación Rodilla - Hemisferio } \\
\text { Izquierdo - Momento Flex }\end{array}$ & 1,058 & 0,613 & 57,889 \\
\hline $\begin{array}{l}\text { Maniobra Pivoting - Articulación Rodilla - Hemisferio } \\
\text { Izquierdo - Momento Ext }\end{array}$ & 0,634 & 0,386 & 60,961 \\
\hline $\begin{array}{c}\text { Maniobra SL leg - Articulación Rodilla - Hemisferio } \\
\text { Derecho - Momento Rot Ext }\end{array}$ & 0,335 & 0,17 & 50,93 \\
\hline
\end{tabular}

En comparación con las mediciones pre, las mediciones del momento rotador interno de la rodilla derecha en la maniobra pivote aumentó la variabilidad alrededor del doble de lo observado mientras que las mediciones del momento flexor de la rodilla derechay el momento extensor de la rodilla izquierda durante la maniobra pivote disminuyeron su variabilidad, esto se puede evidenciar en la Tabla 2.
En la Tabla 3 se observan los resultados del análisis exploratorio para las mediciones post versus pre, en este se evidenció que dichas diferencias presentan rangos de variación muy amplios, la mínima variabilidad se observó para las diferencias de las mediciones del momento extensor de la rodilla izquierda al ejecutar la maniobra pivote. 
TABLA 3. ANÁLISIS EXPLORATORIO MEDICIONES POST VERSUS PRE

\begin{tabular}{|c|c|c|c|}
\hline & \multicolumn{3}{|c|}{ Post vs Pre } \\
\hline Variables & Promedio & Desviación & $\begin{array}{c}\text { Coef. } \\
\text { Variación }\end{array}$ \\
\hline $\begin{array}{c}\text { (Maniobra Pivoting - Articulación Rodilla - } \\
\text { Hemisferio Izquierdo - Momento Ext }\end{array}$ & $-0,527$ & 0,719 & 136,338 \\
\hline
\end{tabular}

TABLA 4. RESULTADOS DEL ANÁLISIS BAYESIANO PARA LAS VARIABLES ANALIZADAS CON MUY FUERTE SOPORTE PARA H1

\begin{tabular}{|c|c|c|c|}
\hline & & $\mu_{\Delta}$ & $\alpha_{1}=P\left(H_{1}: \mu_{\Delta}>0 \mid \Delta_{1}, \Delta_{2}, \ldots\right)$ \\
\hline \multirow{5}{*}{$\begin{array}{l}\text { Flexión rodilla } \\
\text { derecha- Cutting }\end{array}$} & Media & 3,049 & \multirow{5}{*}{1,0000} \\
\hline & Mediana & 3,054 & \\
\hline & Desviación & 0,486 & \\
\hline & \multirow{2}{*}{ RC } & 2,099 & \\
\hline & & 3,993 & \\
\hline \multirow{5}{*}{$\begin{array}{c}\text { Flexión rodilla } \\
\text { izquierda- Cutting }\end{array}$} & Media & 1,327 & \multirow{5}{*}{1,0000} \\
\hline & Mediana & 1,331 & \\
\hline & Desviación & 0,384 & \\
\hline & \multirow{2}{*}{ RC } & 0,575 & \\
\hline & & 2,074 & \\
\hline \multirow{5}{*}{$\begin{array}{c}\text { Flexión rodilla } \\
\text { derecha- Pivoting }\end{array}$} & Media & 4,236 & \multirow{5}{*}{1,0000} \\
\hline & Mediana & 4,241 & \\
\hline & Desviación & 0,469 & \\
\hline & \multirow{2}{*}{ RC } & 3,318 & \\
\hline & & 5,145 & \\
\hline \multirow{5}{*}{$\begin{array}{c}\text { Flexión rodilla } \\
\text { izquierda- Pivoting }\end{array}$} & Media & 2,003 & \multirow{5}{*}{1,0000} \\
\hline & Mediana & 2,009 & \\
\hline & Desviación & 0,553 & \\
\hline & \multirow{2}{*}{ RC } & 0,921 & \\
\hline & & 3,076 & \\
\hline
\end{tabular}

TABLA 5. RELACIÓN DE SOPORTE PARA LA H1 RESPECTO A LA DOMINANCIA Y AÑOS DE EXPERIENCIA EN VARIABLE DE FLEXIÓN MEDIDA EN MANIOBRAS DE CORTE Y PIVOTE

\begin{tabular}{|l|c|c|c|c|c|}
\hline \multicolumn{1}{|c|}{ FLEXION } & $\begin{array}{c}\text { Muy fuerte soporte } \\
\text { para H1 en } \\
\text { futbolistas de } \\
\text { dominancia derecha }\end{array}$ & $\begin{array}{c}\text { Muy fuerte soporte } \\
\text { para H1 en } \\
\text { futbolistas de } \\
\text { dominancia izquierda }\end{array}$ & $\begin{array}{c}\text { Muy fuerte soporte } \\
\text { para H1 en grupo de } \\
\text { futbolistas entre 0-4 } \\
\text { años de experiencia }\end{array}$ & $\begin{array}{c}\text { Muy fuerte soporte } \\
\text { para H1 en grupo de } \\
\text { futbolistas entre 4-8 } \\
\text { años de experiencia }\end{array}$ & $\begin{array}{c}\text { Muy fuerte soporte } \\
\text { para H1 en grupo de } \\
\text { futbolistas con mas de } \\
8 \text { años de experiencia }\end{array}$ \\
\hline Cutting rodilla derecha & 2,889 & $*$ & 3,115 & $*$ & 0,866 \\
\hline Cutting rodilla izquierda & 0,939 & $*$ & 2,122 & $*$ & 2,967 \\
\hline Pivoting rodilla derecha & 5,397 & $*$ & $*$ & 10,06 & 4,097 \\
\hline Pivoting rodilla izquierda & $*$ & 2,176 & $*$ & $*$ & 8,89 \\
\hline Single landing leg rodilla izquierda & $*$ & $*$ & $*$ & & $*$ \\
\hline
\end{tabular}

*Variable medida en la maniobra no cumplió los parámetros de la hipótesis alterna 
TABLA 6. RESULTADOS DEL ANÁLISIS BAYESIANO PARA LAS VARIABLES ANALIZADAS CON DÉBIL SOPORTE PARA H1.

\begin{tabular}{|c|l|c|}
\hline MANIOBRA & \multicolumn{1}{|c|}{ VARIABLES } & \multicolumn{1}{|c|}{ RC } \\
\hline \multirow{5}{*}{ Pivoting } & momento flexor rodilla izquierda & $-0,245$ \\
\cline { 2 - 3 } & momento flexor rodilla derecha & $-0,198$ \\
\cline { 2 - 3 } & momento rotador interno rodilla derecha & $-0,084$ \\
\cline { 2 - 3 } & momento rotador externo rodilla derecha & $-0,093$ \\
\cline { 2 - 3 } & momento rotador externo rodilla izquierda & $-0,047$ \\
\cline { 2 - 3 } & momento adductor rodilla derecha & $-0,182$ \\
\cline { 2 - 3 } & momento adductor rodilla izquierda & $-0,169$ \\
\hline \multirow{4}{*}{\begin{tabular}{c} 
Cutting \\
\cline { 1 - 3 }
\end{tabular}} & momento flexor rodilla derecha & $-0,246$ \\
\cline { 2 - 3 } & momento flexor rodilla izquierda & $-0,222$ \\
\cline { 2 - 3 } & momento extensor rodilla izquierda & $-0,441$ \\
\cline { 2 - 3 } & momento adductor rodilla izquierda & $-0,138$ \\
\cline { 2 - 3 } & momento adductor rodilla derecha & $-0,102$ \\
\cline { 2 - 3 } & momento rotador externo rodilla izquierda & $-0,051$ \\
\cline { 2 - 3 } & momento rotador externo rodilla derecha & $-0,059$ \\
\hline \multirow{2}{*}{ Single } & momento rotador externo rodilla derecha & $-0,055$ \\
\cline { 2 - 3 } & momento rotador externo rodilla izquierda & $-0,057$ \\
\hline
\end{tabular}

En la Tabla 4 se evidenció los resultados del análisis bayesiano para las variables que mayor soporte mostraron para la hipótesis alterna $(H 1: \mu \Delta>0)$, donde se estableció que dichas variables tienen mayor probabilidad de presentar valores post mayores en comparación con los valores pre.

Según la hipótesis planteada se espera que existan cambios cuando hay presencia de fatiga al ejecutar las maniobras establecidas, se evidenció por lo tanto que hubo cambios en las variables de flexión y extensión de rodilla cumpliendo la hipótesis alterna donde se estableció que las medidas post son mayores que las medidas pre, cumpliendo con la condición $\alpha 1=P(H 1$ : $\mu \Delta>0 \mid \Delta 1, \Delta 2, \ldots)$, donde $\mathrm{RC}>0$. Esto se pudo evidenciar principalmente en las siguientes variables, en diferentes situaciones de puntos de comparación:

Sobre la dominancia de los futbolistas, se encontró que el 74.2\% (23 futbolistas) son de dominancia derecha.

En la Tabla 6 se evidenció el comportamiento de las variables asociadas a los momentos abductores, aductores y los momentos rotadores internos y externos, donde no hubo cambios significativos entre las condiciones pre y post fatiga, ya que no se cumplió con la condición de la hipótesis alterna. Por lo tanto, se puede determinar que para estas variables hubo un débil soporte para $\mathrm{H} 1$.

\section{Discusión}

El objetivo principal de la investigación fue describir los efectos de la fatiga muscular en miembros inferiores, específicamente y de manera preliminar enfocada a la biomecánica de la rodilla, siendo esta de las principales articulaciones involucradas en la lesión de ligamento cruzado anterior. Para llevar a cabo esta descripción se llevó a cabo diversas evaluaciones usando diferentes maniobras típicas de fútbol que incluyen cambios de dirección, desaceleraciones y aterrizajes en una sola pierna, ya que estas condiciones son consideradas como factores de riesgo en el mecanismo de lesión por no contacto, adicionalmente, fue importante considerar el factor fatiga ya que hay mayor incidencia de la lesión observada en la segunda mitad de un partido competitivo, especialmente en los últimos quince minutos (Cruz, 2013; Hawkins, 2001).

El principal hallazgo de la investigación fue que no se presentaron cambios significativos en las variables 
cinemáticas medidas en el plano frontal como aducción de rodilla, teniendo en cuenta que durante la recolección de los datos no hubo presencia de ángulos de abducción en las rodillas derecha e izquierda en los deportistas, tampoco hubo cambios significativos en las variables cinemáticas en el plano transverso como rotación interna y externa en presencia de fatiga en la maniobra de aterrizaje en una sola pierna, de manera similar y determinando que la cinemática del plano frontal, se ha demostrado en varios estudios que no hay efectos sobre el valgo de rodilla en tareas como saltos verticales en una sola pierna y aterrizajes de salto en una sola pierna (Benjaminse, 2008; Kernozek, 2008; Liederbach, 2014; Lessi, 2017). [6, 7, 26, 55]. Con respecto a las maniobras de corte y pivote se encontró que tampoco se presentaron cambios significativos respecto a la maniobra de aterrizaje en una sola pierna, similar a esto se puede mencionar que estas tareas se limitaron a condiciones anticipadas, asociándolo al hecho de que los deportistas ya sabían los movimientos que debían llevar a cabo, mientras que en tareas y condiciones no anticipadas se ha demostrado que los deportistas pueden alcanzar mayores demandas cinéticas y cinemáticas (Besier et al., 2001). Por lo tanto, es importante tener en cuenta que si bien la estandarización de una tarea/maniobra no anticipada puede lograrse en un entorno de laboratorio, tal condición puede aumentar las cargas y demandas biomecánicas en dicha articulación, por lo que se puede explicar que la no variación en estas medidas se debe a condiciones anticipadas de los deportistas al llegar a la plataforma de fuerza y realizar los giros.

Contrario a lo que se ha mencionado, la variación en la flexión de la rodilla en ambas piernas se vio afectada moderadamente, es decir las mediciones post alcanzaron el doble del valor respecto a la medición pre en todas las maniobras ejecutadas, esto se puede contrastar con lo descrito en la revisión sistemática realizada por Serpell, donde encontraron que el efecto de la fatiga en la cinemática de la rodilla se estudió en varios estudios de laboratorio y los resultados variaron. Sin embargo, en dos estudios demostraron que hubo un incremento en el ángulo de flexión de la rodilla en toda la fase de contacto en tareas de pivote y aterrizaje de un salto o pivote (Serpell et al., 2012), de lo obtenido en esta investigación hay una fuerte correspondencia en la maniobra del aterrizaje.
Es posible determinar que en muchas ocasiones se podría asociar el aumento del ángulo de flexión con poco control del movimiento debido a la presencia de la fatiga, pero en la investigación de Sugimoto et al (2015), mencionan que el aumento en la flexión de rodilla en la fase de aterrizaje puede estar involucrada con menor riesgo de lesión de ligamento cruzado anterior, esto debido a su hallazgo el cual refiere que si hay aterrizajes con mayor extensión de rodilla, este se asocia directamente con un mayor riesgo de lesión, ya que los músculos actúan como estabilizadores dinámicos, los cuales se modifican de acuerdo a la posición articular.

En las variables cinéticas como los momentos de fuerza en la articulación sobre los tres ejes de movimiento, no se evidenciaron cambios significativos después de la fatiga generando débil soporte para la hipótesis alterna. De hecho, en la maniobra de pivote se presentó débil soporte en una mayor cantidad de variables en comparación con las maniobras de corte y aterrizaje de un salto en una sola pierna de ambos miembros inferiores. Esto se puede relacionar con lo encontrado por Barber-Westin donde refiere no hay diferencias uniformes entre tareas planeadas (anticipadas) y tareas reactivas (no anticipadas) respecto a efectos de la postfatiga en las variables cinemáticas y cinéticas de los miembros inferiores.

El momento abductor de la rodilla no tuvo soporte con la hipótesis alterna, lo cual se pudo evidenciar en el hecho de que ningún deportista presentó ángulos de abducción de rodilla, concordando esto con lo encontrado por Hewett et al. (2005) donde reportó que esta variable de la rodilla es un mejor predictor de la lesión de ligamento cruzado anterior en deportistas femeninas. De la revisión sistemática más reciente al considerar las cargas en la articulación de la rodilla, se demostró que no hay efecto significativo de la fatiga en los momentos de abducción en las tareas de salto y pivote (Benjaminse 2019). Otras variables son el momento extensor en plano sagital y momentos de rotación externa e interna, las cuales presentaron variaciones entre las pruebas pero no aplicaron a las condiciones de la hipótesis alterna al no presentar cambios significativos en la presencia de fatiga, tal como lo reporta Kernozek et al., (2008) que los hombres y las mujeres presentaron momentos extensores significativamente bajos siguiendo un protocolo de fatiga. 
Es importante destacar que aunque el modelo biomecánico utilizado incluye articulaciones de cadera y tobillo, el enfoque de la investigación está puesto en la articulación de la rodilla, se recomienda en futuras investigaciones realizar comparaciones del comportamiento de tales articulaciones incluyendo pelvis y tronco ya que se ha demostrado que la influencia de la fatiga en la posición del tronco es un aspecto importante debido a que esto puede cambiar las cargas en la articulación de la rodilla y el estrés en la lesión de ligamento cruzado anterior (Kulas et al. 2012).

Según los hallazgos y diferencias de la investigación según los resultados obtenidos, parece que el protocolo de fatiga implementado en los jugadores de fútbol juveniles no representó una intensidad de ejercicio lo suficientemente alta como para fatigar por completo el deportista, según concentraciones de lactato en músculo, de acuerdo también al esfuerzo percibido por cada uno y una disminución evidente del control muscular como lo indica Lehnert et al., (2018) en su investigación.

Una gran limitante de la investigación se enfoca en determinar si el protocolo de fatiga aplicado fue el adecuado para producir los cambios significativos esperados en las variables cinemáticas y cinéticas de la articulación de la rodilla, ya que en la investigación de Santamaría et a.l (2010), determinaron que no hay establecido un método consistente para asegurar un adecuado protocolo que induzca fatiga de manera exitosa, en una población determinada, lo cual puede verse reflejado en un entendimiento incompleto de la mecánica de la fatiga, en particular, fatiga neuromuscular. También se recomienda tener en cuenta la medición de las fuerzas de reacción verticales al suelo medidas en cada maniobra en ambos miembros inferiores para tener una relación y comparación de tales variables con los momentos de fuerza que se generaron en la rodilla, contrastando esto con lo referido por Hewett et al., (2005) que refiere que hay evidencia que sugiere que las fuerzas verticales más elevadas pueden aumentar el riesgo de lesión de ligamento cruzado anterior durante este tipo de maniobras. Sin embargo, también sugerir que los atletas fatigados en realidad se moverían de forma más lenta y esto generaría que se produzcan menos fuerzas de reacción en el suelo que en deportistas no fatigados (Bourne et al., 2007).

\section{Conclusión}

Los resultados de esta investigación mostraron que las variables cinemáticas del plano sagital se vieron principalmente afectadas después de la fatiga en todas las maniobras realizadas en ambos miembros inferiores. Sin embargo, el protocolo de fatiga implementado no generó un efecto significativo en la mayoría de las variables de los planos frontal y transverso, a su vez las variables cinéticas tampoco se vieron afectadas. El poco efecto generado en las variables analizadas en esta investigación resalta la necesidad de una investigación continua en el mejoramiento de la evaluación y monitoreo de la fatiga para relacionar con la incidencia de una lesión como la del ligamento cruzado anterior. En efecto, se considera que las maniobras evaluadas sí involucran movimientos asociados a mecanismo de lesión por no contacto, pero es importante analizar la biomecánica de las demás articulaciones involucradas pues se evidencia en diferentes estudios que hay mayores cambios variables cinemáticas de la cadera y tobillo. Es importante considerar aspectos como el nivel de esfuerzo percibido en el jugador y tener en cuenta los mecanismos de anticipación en cuanto a las maniobras establecidas, determinar si este tipo de factores también pueden ser un factor incidente en la modificación del comportamiento de algunas variables que puedan estar involucradas en el riesgo de lesión de ligamento cruzado anterior. Finalmente, la prevención de lesiones como área de investigación debe extenderse a profesionales del desempeño deportivo pues tendría bases para mejorar planes de entrenamiento más específicos y también para la rehabilitación deportiva pues la probabilidad de recurrir en la lesión es alta y aún más cuando la fatiga puede desempeñar un papel importante para el retorno del deportista a la práctica deportiva.

\section{Conflictos de interés}

Los autores manifiestan que no hay conflictos de interés asociados a la presente investigación.

\section{Agradecimientos}

Los autores reconocen y agradecen la contribución que realizó Qualisys con la tecnología para el desarrollo de la investigación y a la Institución 
Universitaria Escuela Nacional de Deporte (IUEND) por las instalaciones. Además, extender agradecimiento a las directivas del club deportivo y sus deportistas por su disposición.

\section{Referencias}

Hägglund M., Waldén M., Magnusson H., Kristenson K., Bengtsson H., Ekstrand J. Injuries affect team performance negatively in professional football: an 11-year follow-up of the UEFA Champions League injury study. Br J Sports Med, 47(12),738-42, 2013.

Ali A., Williams C., Nicholas CW., Foskett A. The influence of carbohydrate-electrolyte ingestion on soccer skill performance. Med Sci Sports Exerc, 39,1969-1976, 2007.

Ostojic S., Mazic S. Effects of a carbohydrate electrolyte drink on specific soccer tests and performance. J Sports Sci Med, 2,47-53, 2002.

Parra C. La lesión del ligamento cruzado anterior en el fútbol femenino. Tesis de grado. Ciencias de la actividad física y del deporte, Universidad Politécnica de Madrid, 2014.

Zahínos J., González C., Salinero J. Estudio epidemiológico de las lesiones. Los procesos de adaptación y prevención de la lesión de ligamento cruzado anterior en el fútbol profesional.Journal of Sport and Health Research, 2(2), 139-150, 2010.

Boden BP., Dean GS., Feagin JA. Mechanisms of anterior cruciate ligament injury. Orthopedics, 23(6), 573-578, 2007.

Brophy R., Silvers HJ., Gonzales T., Mandelbaum BR. Gender influences: The role of leg dominance in ACL injury among soccer players. British Journal of Sports Medicine, 44(10), 694-697, 2010.

Rodacki ALF., Fowler NE., Bennett SJ. Multi-segment coordination: Fatigue effects. Medicine and science in sports and exercise, 33(7), 1157-1167, 2001.

Padua DA., Arnold BL., Perrin DH., Gansneder BM., Carcia CR., Granata KP. Fatigue, vertical leg stifness, and stifness control strategies in males and females. J Athl Train, 41(3), 294-304, 2006.

Barrett S., Lovell R., Guard A. SAFT90 simulates the internal and external loads of competitive soccer match-play. Proceedings of the Seventh World Congress on Science and Football, Liverpool, 2013.

Jones PA., Herrington LC., Munro, AG., Graham-Smith P. Is there a relationship between landing, corte, and pivote tasks in terms of the characteristics of dynamic valgus?. The American Journal of Sports Medicine, 42(9), 2095-102, 2014.
Winter DA. Biomechanics and motor control of human movement. John Wiley and Sons, Inc; New York: 1990. Capítulos 1,2,3, 5 .

Small, K., McNaughton, L. R., Greig, M., Lohkamp, M., \& Lovell, R. Soccer fatigue, sprinting and hamstring injury risk. International Journal of Sports Medicine, 30(8), 573-578, 2009.

Cruz A., Bell D., McGrath M., Blackburn T., Padua D. \& Herman D.The Effects of Three Jump Landing Tasks on Kinetic and Kinematic Measures: Implications for ACL Injury Research, Research in Sports Medicine: An International Journal, 21:4,330-342, 2013.

Hawkins, R., Hulse, M., Wilkinson, C., Hodson, A., \& Gibson, M., The association football medical research programme: an audit of injuries in professional football. British Journal of Sports Medicine, 35(1), 43-47, 2001.

Benjaminse A, Habu A, Sell TC, Abt JP, Fu FH, Myers JB., Fatigue alters lower extremity kinematics during a single-leg stop-jump task. Knee Surg Sports Traumatol Arthrosc.;16(4), 400-7, 2008.

Kernozek TW, Torry MR, Iwasaki M. Gender differences in lower extremity landing mechanics caused by neuromuscular fatigue, Am J Sports Med; 36 (3), 554-65, 2008.

Liederbach M, Kremenic IJ, Orishimo KF, Pappas E, Hagins M. Comparison of landing biomechanics between male and female dancers and athletes, part 2: influence of fatigue and implications for anterior cruciate ligament injury, Am J Sports Med; 42 (5), 1089-95, 2014.

Lessi GC, Dos Santos AF, Batista LF, de Oliveira GC, Serrao FV., Effects of fatigue on lower limb, pelvis and trunk kinematics and muscle activation: gender differences, J Electromyogr Kinesiol.; 32, 9-14, 2017.

Besier, T. F., Lloyd, D. G., Ackland, T. R., \& Cochrane, J. L., Anticipatory effects on knee joint loading during running and corte maneuvers, Medical Science and Sports Exercise, 33 (7), 1176-1181, 2001.

Serpell, B. G., Scarvell, J. M., Ball, N. B., \& Smith, P. N., Mechanisms and Risk Factors for Noncontact ACL Injury in Age Mature Athletes Who Engage in Field Or Court Sports, Journal of Strength and Conditioning Research, 26 (11), 3160-3176, 2012.

Sugimoto D, Alentorn-Geli E, Mendiguchía J, Samuelsson K, Myer GD., Biomechanical and Neuromuscular Characteristics of Male Athletes: Implications for the Development of Anterior Cruciate Ligament Injury Prevention Programs, Sports Medicine Journal, 45 (6), 809-22, 2015.

Barber-westin, S.D, Noyes, B.S \& Noyes, F.R., Effect of Fatigue Protocols on Lower Limb Neuromuscular Function and Implications for Anterior Cruciate Ligament 
Injury Prevention Training: A Systematic Review. The American Journal of Sports Medicine, 1(1),1 - 9, 2017.

Hewett TE, Myer GD, Ford KR, Heidt RS, Colosimo AJ, McLean SG, Biomechanical measures of neuromuscular control and valgus loading of the knee predict anterior cruciate ligament injury risk in female athletes: a prospective study, Am J Sports Med, 33(4), 492-501, 2005.

Benjaminse A., Webster KE., Kimp A., Meijer M., Gokeler A. Revised approach to the role of fatigue in anterior cruciate ligament injury prevention: a systematic review with meta-analyses. Sports Med, 49(4), 565-86, 2019.

Kulas AS., Hortobágyi T., DeVita P. Trunk position modulates anterior cruciate ligament forces and strains during a single-leg squat. Clin Biomech, 27(1), 16-21, 2012.

Lehnert M., Croix MDS., Xaverova Z., Botek M., Varekova R., Zaatar A., Stastny P. Changes in Injury Risk Mechanisms After Soccer-Specific Fatigue in Male Youth Soccer Players. Journal of Human Kinetics, 62(1), 33-42, 2018.

Santamaria LJ., Webster KE. The effect of fatigue on lowerlimb biomechanics during single-limb landings: a systematic review. Journal of orthopaedic and sports physical therapy, 40(8), 464-73, 2010.

Bourne MN., Webster KE., Hewett TE. Is fatigue a risk factor for anterior cruciate ligament rupture? Sports Medicine, 49(11), 1629-1635, 2019.
PARA CITAR ESTE ARTÍCULO / TO REFERENCE THIS ARTICLE / PARA CITAR ESTE ARTIGO /

Quintero Palma, L.A.; Agredo, W.; Quiceno Henao, J.; Quiñones, A.; Tovar, J.F. (2020). Efectos de fatiga en variables cinemáticas y cinéticas de miembros inferiores en jugadores de fútbol. Revista EIA, 17(33) enerojunio, Reia33018 pág. 1-13. Disponible en: https://doi. org/10.24050/reia.v17i33.1360 